\title{
X-ray Attenuation and Reduction of Backscattered Radiation
}

\author{
A. T. Naji ${ }^{1}$, M. S. Jaafar ${ }^{1}$, E. A. Ali ${ }^{2} \&$ S. K. J. Al-Ani ${ }^{3}$ \\ ${ }^{1}$ School of Physics, University of Science Malaysia (USM), Pulau Pinang, Malaysia \\ ${ }^{2}$ College of Engineering, University of Science and Technology, Sana'a, Yemen \\ ${ }^{3}$ Department of Physics, College of Science, University of Baghdad, Baghdad, Iraq \\ Correspondence: S. K. J. Al-Ani, Department of Physics, College of Science, University of Baghdad, Baghdad, \\ Iraq. E-mail: salwan_kamal@yahoo.com
}

Received: June 27, 2016

Accepted: July 9, 2016

Online Published: July 29, 2016

doi:10.5539/apr.v8n4p92

URL: http://dx.doi.org/10.5539/apr.v8n4p92

\begin{abstract}
This paper reports the comparative study of X-ray attenuation using different materials which can be utilized to design anti-backscattered grids and radiation protection shields. The characteristics of X-ray attenuation in several materials, namely, lead, copper, iron steel, and aluminum, are investigated under diverse exposure parameters to measure their ability to attenuate incident X-ray and their capability to reduce backscattered X-ray. Lead and iron steel shields exhibit the best abilities in attenuating X-ray, whereas aluminum shield shows the least attenuation ability. This study proposes a design of a unique method for assessing the backscatter X-ray dose. The iron steel grid based on iron steel plate provides the best value in reducing backscattered radiation (up to $34.78 \%$ ) compared with other conventional plate materials.
\end{abstract}

Keywords: attenuation of X-ray, backscattered radiation, incident X-ray, metals, shield

\section{Introduction}

Medical X-ray is widely used for clinical diagnosis and considered a major artificial source of ionizing radiation received by patients, staff, and the public (Huggett et al., 2013). Many surveys have shown a wide distribution of doses for patients' requiring the same type of radiographic examination (Kim et al., 2012). Skin diseases, thyroid cancer, brain tumors, and cataracts are several examples of the adverse effects of ionizing radiation on the human body (Ryu et al., 2013).

Exposure of medical personnel to X-ray can persist for as long as several hours. Thus, a new generation of protective devices should be developed to provide reliable protection for medical personnel against X-ray radiation (Blinov, 2013). Attenuation of X-ray beams depends on several exposure parameters, such as applied potential voltage $(\mathrm{kVp})$, intensity of radiation exposure (mAs), and focal spot- detector distance (FDD). These parameters should be selected accurately according to the medical indication (Aichinger et al., 2012).

Pure metals exhibit more effective attenuation than materials with powdered metals embedded in a polymer matrix. This matrix adds weight to the material but has poor attenuation capability. The capability of a shield material in attenuating radiation depends on the metal density and atomic number (McCaffrey et al., 2012).

Available radiation protection methods using concrete and lead shields provide suitable protection for personnel in the radiographer room (control room). However, backscattered radiation protection for a patient and the technician inside the X-ray room during radiography, fluoroscopy, and interventional radiology procedures still require further optimization and investigation.

This study was performed to investigate a method to reduce the backscattered radiation inside an X-ray room. The capabilities of several shield materials in attenuating the incident and backscattered X-ray were estimated by a comparative study of the different materials, namely, lead, copper, iron steel, and aluminum. These materials may be used to design anti-backscattered grids, X-ray shields, and radiation protection materials. 


\section{Method}

\subsection{Experiment's Materials}

Several shields made from lead, iron steel, copper, and aluminum with different thicknesses are tested. Previous studies investigated half value layers and attenuation coefficients of these metals. By contrast, the properties of these metals in attenuating diagnostic X-ray at different energies and different radiation intensities are investigated in the current study. The effect of variation in metal thickness is also examined to establish the attenuation behavior of these metals at different exposure settings. In addition, the capabilities of these metals in attenuating backscattered radiation are determined for possible application as substitutes to current shields.

\subsubsection{X-ray Unit}

General radiography X-ray is the main source of radiation in this study. The major components of the X-ray generator are the X-ray tube, a high-voltage generator, a controlled console, and a cooling system.

\subsubsection{Ionization Chamber}

An ionization chamber (PTW flat chamber connected to a PTW UNIDOS electrometer) is used to measure the penetrating (incident) and backscattered X-ray dose. Its calibration was consistent with the standards of dosimetry, Malaysian nuclear agency. For measuring penetrated (attenuated) radiation, the ionization chamber is positioned away from the surface of X-ray table to avoid measuring backscattering radiation as it is illustrated in Figure 1-a.

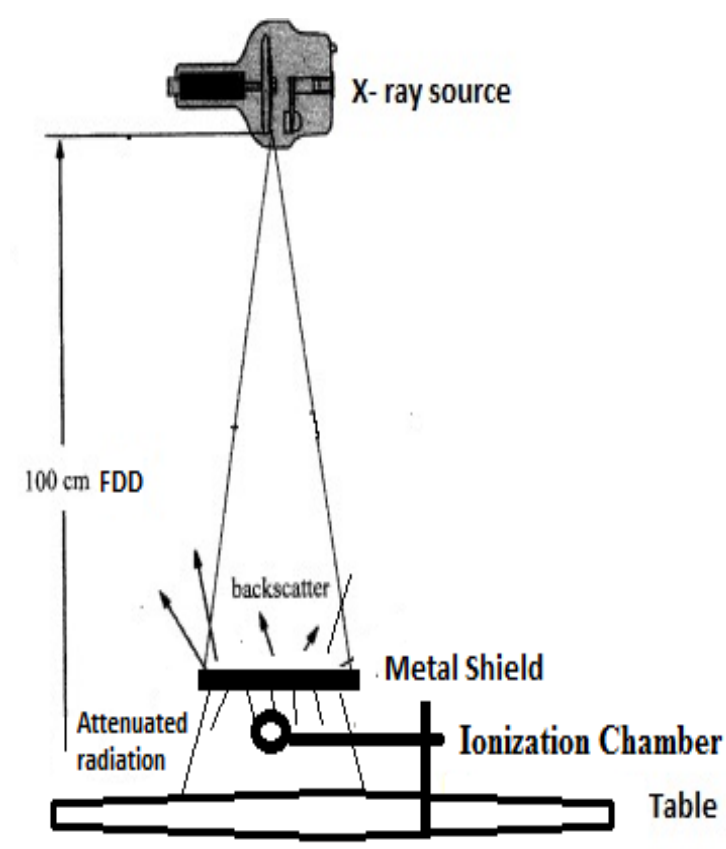

a) Measuring the penetrated radiation

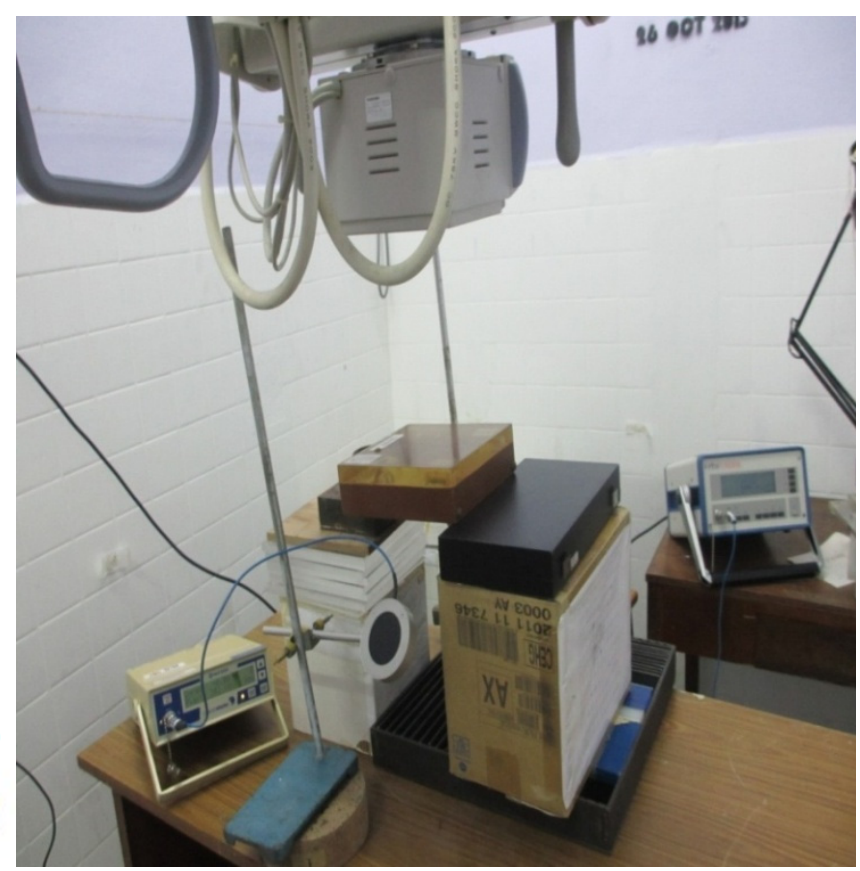

b) Measuring the backscattered radiation

Figure1. The experiments setups

\subsection{Experimental Procedures}

Some investigations are carried out to evaluate the important parameters associated with quality control of the Xray machine as follows: first, check the $\mathrm{kVp}$ value using the $\mathrm{kVp}$ and HVL test tool (Wisconsin X-ray Test Cassette, Figure A in Appendix) to determine the accuracy of the kVp setting for X-ray machine. Second, check the X-ray timer by utilizing Timing and mAs test tool (RMI Model 121A, Figure B in Appendix) to determine whether the X-ray output is constant for a given $\mathrm{mAs}$ when different $\mathrm{mA}$ values are used. Third, determine the focal spot size by using focal spot test tool (RMI Model 112B, Figure C in Appendix) to determine the dimension of the effective focal spot size as well as the geometric unsharpness.

The actual results of the investigations are in the range of acceptable values range within $\pm 5 \%$ of the nominal values. These results are in agreement with the quality control in radiography based on Malaysian standards practice for radiation protection and medical X-ray diagnosis (Abdulaziz, 2012). 
Then, the X-ray radiation is directly focused to the different metal plates with different thicknesses to measure their capabilities in attenuating radiation by measuring the penetrating X-ray. The penetrating radiation doses are measured by the flat ionization chamber with an electrometer. The flat ionization chamber is positioned directly behind the tested metal. The measurements are recorded at different exposure parameters, namely, exposure time, $\mathrm{X}$-ray tube current, field size of the X-ray beam, and X-ray tube applied voltage.

Backscattered radiation is measured by placing the flat ionization chamber outside the exposed area and fixed at backscattered angle of $45^{\circ}$ with central ray of incident radiation to measure the backscattered radiation only (Figure $1-b)$.

The measurements of this study have been carried out in the Radiation Physics laboratory, School of Physics, University of Science Malaysia (USM), Malaysia.

\section{Results and Discussion}

The results are divided into two sections: The capability of the tested metals on attenuating the incident X-ray will be discussed in the first section. The second section will report the effect of these metal plates on reducing the backscattered radiation using these plates as a base underlying a backscattered iron steel grid.

\subsection{Capabilities of Tested Metals in X-ray Attenuation}

\subsubsection{X-ray Attenuation using Lead Shields}

The effectiveness of lead shield $\left(\mathrm{Z}=82\right.$, mass density $\left.=11.34 \mathrm{~g} / \mathrm{cm}^{3}\right)$ in attenuating the incident X-ray at different values of applied potential voltage $(\mathrm{kVp})$ ranges from $40 \mathrm{kVp}$ to $120 \mathrm{kVp}$. The readings are recorded under the following conditions: tube current I of $320 \mathrm{~mA}$; exposure time ( $\mathrm{t}$ ) of $0.1 \mathrm{~s}$; and focal spot-detector distance (FDD) of $100 \mathrm{~cm}$. Figure 2 illustrates the comparison of the incident X-ray doses with and without a lead shield.

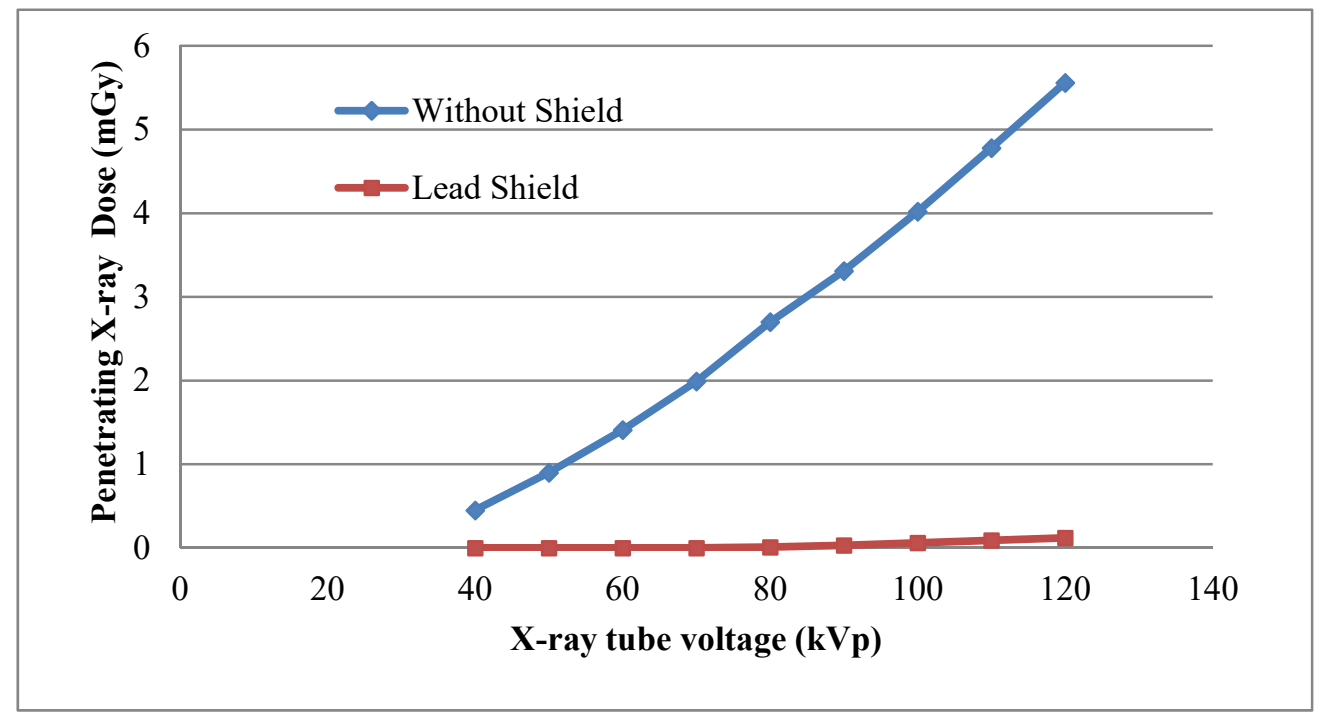

Figure 2. X-ray attenuation for1 $\mathrm{mm}$ Lead shield

Figure 2 presents the X-ray dose as a function of $\mathrm{kVp}$. The capability of the lead shield in attenuating X-ray at different $\mathrm{kVp}$ values is compared with $\mathrm{X}$-ray dose measured without a shield. Remarkable difference was found in the measured X-ray doses with and without the lead shield. The percentages of the attenuated radiation are calculated to demonstrate the behavior of 1-mm thick lead shield in attenuating X-ray at different $\mathrm{kVp}$ values (Figure 3).

Effect of lead thickness on the reduction of the penetrating X-ray dose:

The effect of increasing lead thickness on the capability of this metal for attenuating X-ray and the behavior of the attenuation- $\mathrm{kVp}$ curves with different lead thicknesses are also examined. Various thicknesses of lead are used as shields for X-ray at different $\mathrm{kVp}$ values. Exposure parameters are $\mathrm{I}=320 \mathrm{~mA}, \mathrm{t}=0.1 \mathrm{~s}$, and $\mathrm{FDD}=100 \mathrm{~cm}$. The relationship between lead shield thickness and percentage of X-ray attenuation is illustrated in Figure 3. 


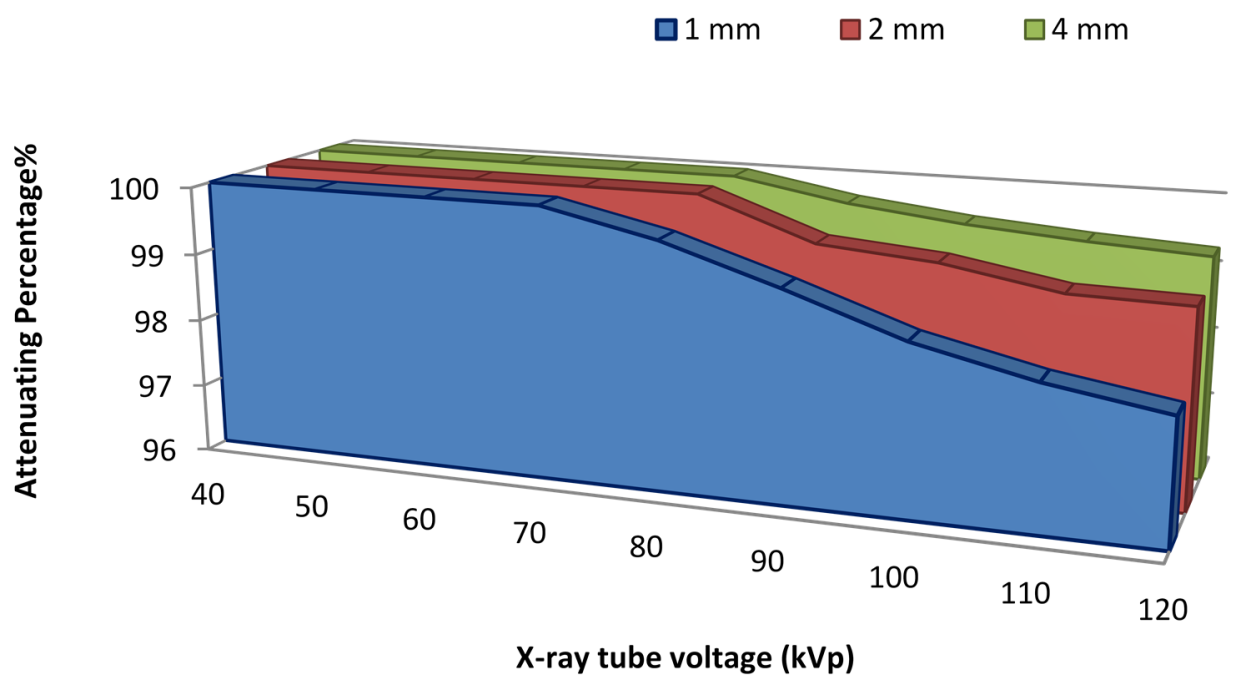

Figure 3. X-ray attenuation for Lead shields with different thicknesses

Figure 3 demonstrates an inverse proportionality between the attenuating radiation by lead shields and $\mathrm{kVp}$. The capability of lead in attenuating X-ray is more significant at lower $\mathrm{kVp}$, and the attenuation percentage of 1-mm thick lead reaches $100 \%$. The percentage of X-ray attenuation by $1-\mathrm{mm}$ thick lead is more than $97.5 \%$ at higher $\mathrm{X}$ - ray energy $(120 \mathrm{kVp})$. Thus, the penetration of the X-ray through lead shield increases with increasing applied voltage of X-ray tube.

In summary, X-ray attenuation depends on the thickness of the lead shield. The penetration of the X-ray through lead shields decreases by increasing lead thickness. The curve of attenuation percentage $-\mathrm{kVp}$ behavior depends on the lead thickness. Decreasing the thickness of lead shield $(1 \mathrm{~mm})$ results in steeper slope of the curve. Moreover, the percentage of penetrated X-ray increases rapidly compared with thicker lead shields. Hence, the attenuation properties for lead have non-linear relation with lead thickness against radiation energy.

\subsubsection{X-ray Attenuation using Copper Shields}

The capability of copper metal $\left(Z=29,8.92 \mathrm{~g} / \mathrm{cm}^{3}\right)$ in attenuating X-ray is examined at exposure parameters of $32 \mathrm{mAs}$ and FDD of $100 \mathrm{~cm}$. Figure 4 shows the recorded X-ray dose and a comparison between the penetrating dose using a copper shield and incident dose without a shield.

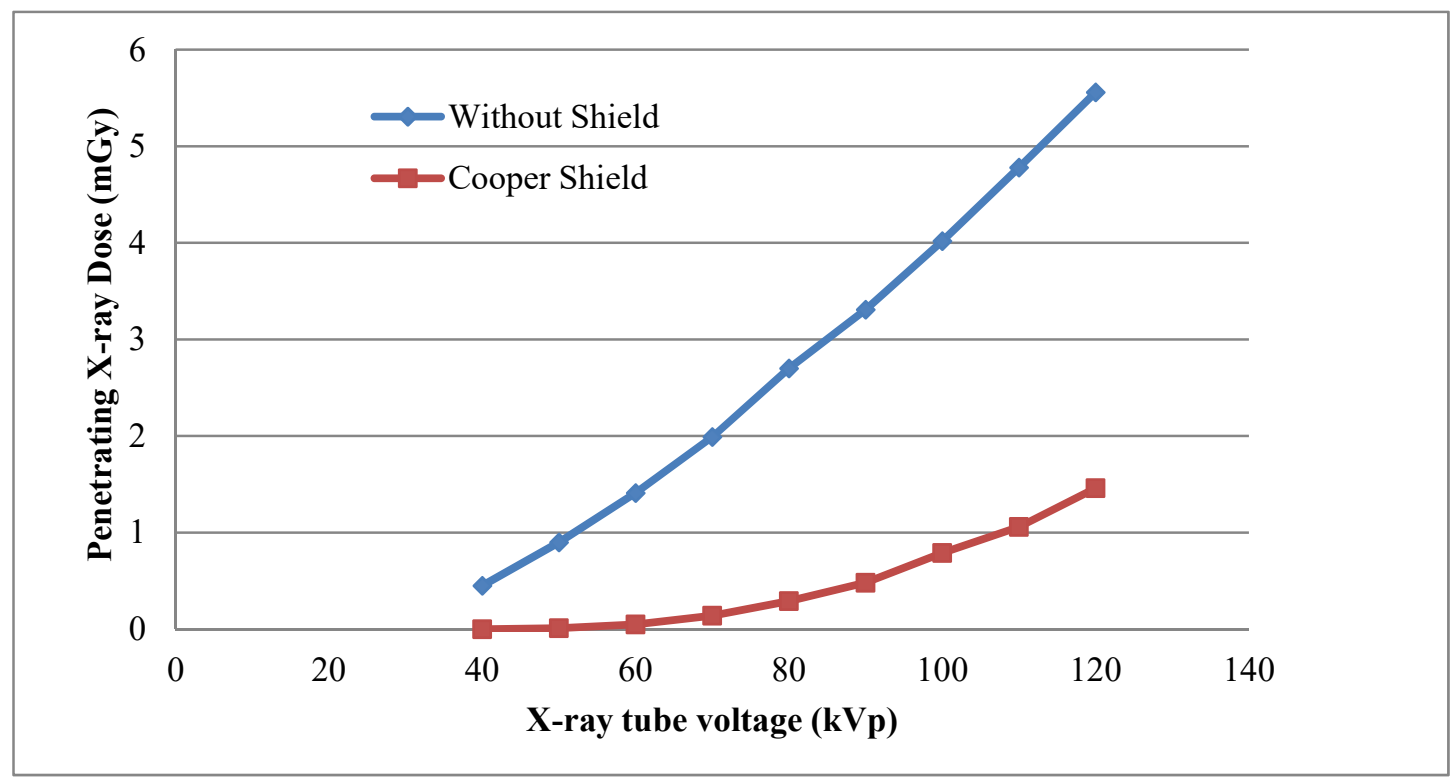

Figure 4. Capability of $1 \mathrm{~mm}$ Copper in attenuation of X-ray 
The penetration of X-ray photons increases by increasing the photons energy. The capability of copper shield in attenuating $\mathrm{X}$ - radiation dose is more significant particularly at lower applied voltage. More X-ray penetrates through $1 \mathrm{~mm}$ copper shield as shown by the high photon energy $(120 \mathrm{kVp})$.

The effectiveness of copper shield thickness on attenuating incident X-ray was examined. Different thicknesses of copper shield are tested at different $\mathrm{kVp}$ values to assess the ability of copper in reducing the penetrated dose. Additionally, the percentages of radiation reduction are calculated and shown in Figure 5.

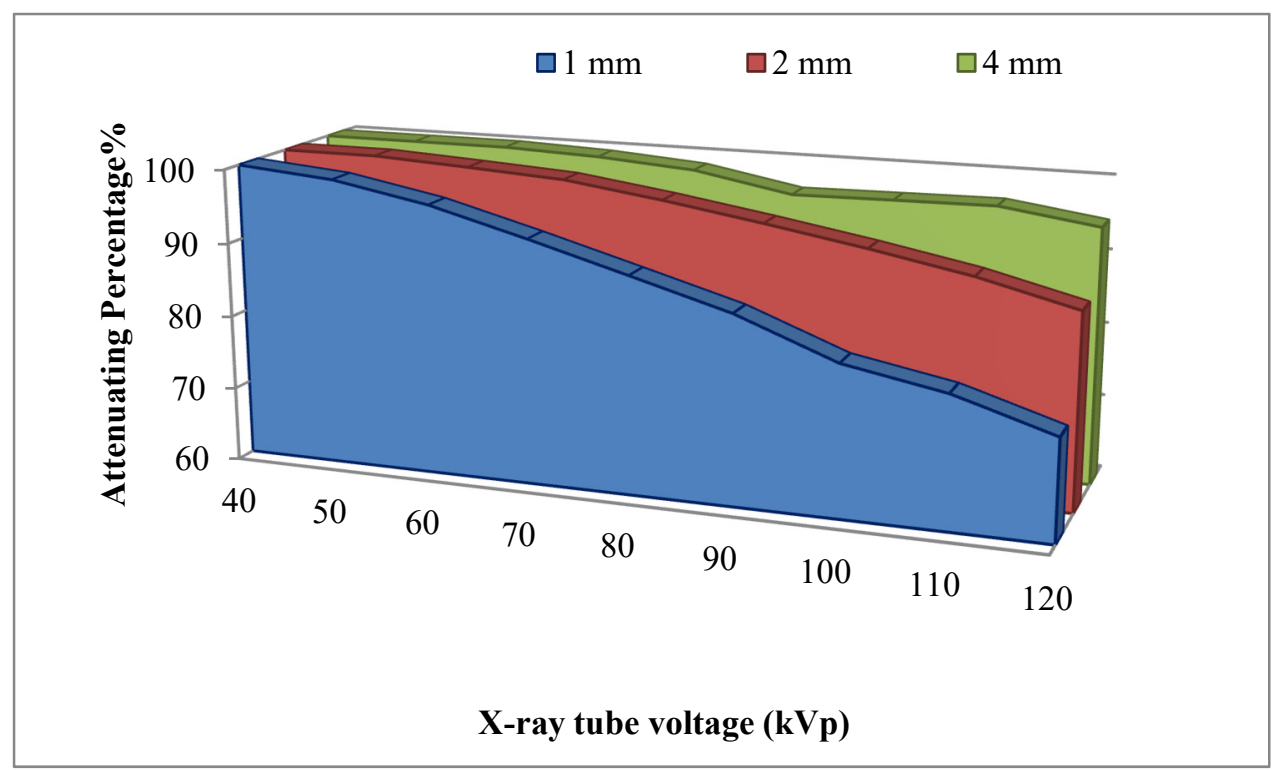

Figure5. The effect of Copper thickness on attenuation of X-ray

The percentage of radiation reduced by $1 \mathrm{~mm}$-thick of copper shield is more than $73 \%$ for all $\mathrm{kVp}$ values. Furthermore, the percentage of X-ray attenuation is directly proportional with copper thickness. Figure 5 shows the effect of copper shield thicknesses on the capability of copper to attenuate the incident X-ray. The percentages of penetrated X-ray increase linearly with $\mathrm{kVp}$. The drop in attenuating percentages by increasing $\mathrm{kVp}$ is steeper for lower copper thickness $(1 \mathrm{~mm})$. Thus, X-ray penetrating through copper shield increases with increasing photon energy and decreasing copper thickness.

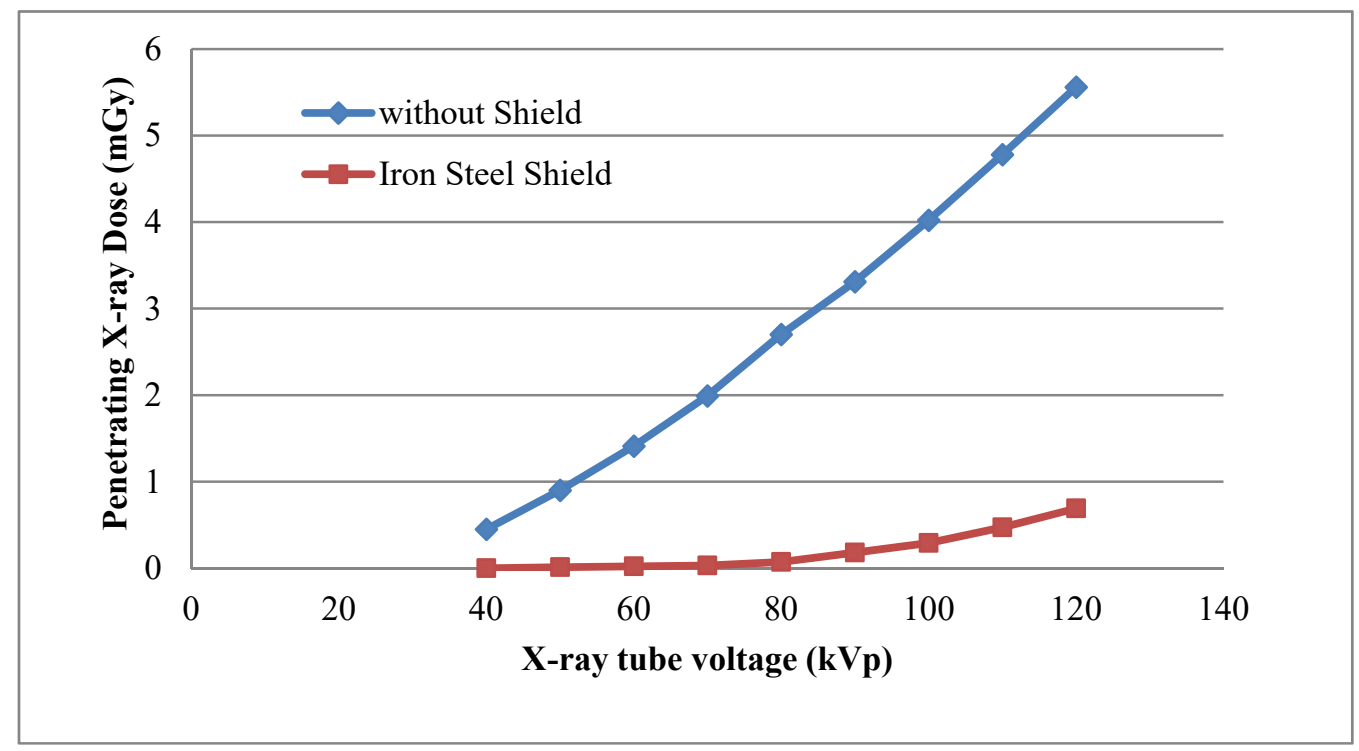

Figure 6. The capability of Iron Steel in attenuating the X-ray 


\subsubsection{X-ray Attenuation using Iron Steel Shields}

The effectiveness of iron steel plate $\left(Z=26\right.$, mass density $\left.=7.874 \mathrm{~g} / \mathrm{cm}^{3}\right)$ in attenuating X-ray is measured. The readings are recorded at $32 \mathrm{mAs}, \mathrm{FDD}$ of $100 \mathrm{~cm}$, and different $\mathrm{kVp}$ values to show the difference between measuring dose with and without iron steel plate as shield against $\mathrm{X}$-ray.

The effectiveness of iron steel shields in reducing the penetrating radiation dose is more significant particularly at low $\mathrm{kVp}$, reaching total attenuation. The attenuation capability of iron is higher than that of copper because of the large mass density of copper. However, the probability of photo scattering effect is directly proportional to the mass density of the attenuating material. This characteristic will affect the total radiation attenuation of copper. Hence, regardless of the atomic number-related photoelectric effect, more X-ray photons are attenuated in iron than in copper.

The effect of iron thickness on attenuating the incident $\mathrm{X}$-ray at different $\mathrm{kVp}$ values and various thicknesses of iron shields are investigated. The attenuation percentages for shields are also calculated and illustrated in Figure 7.

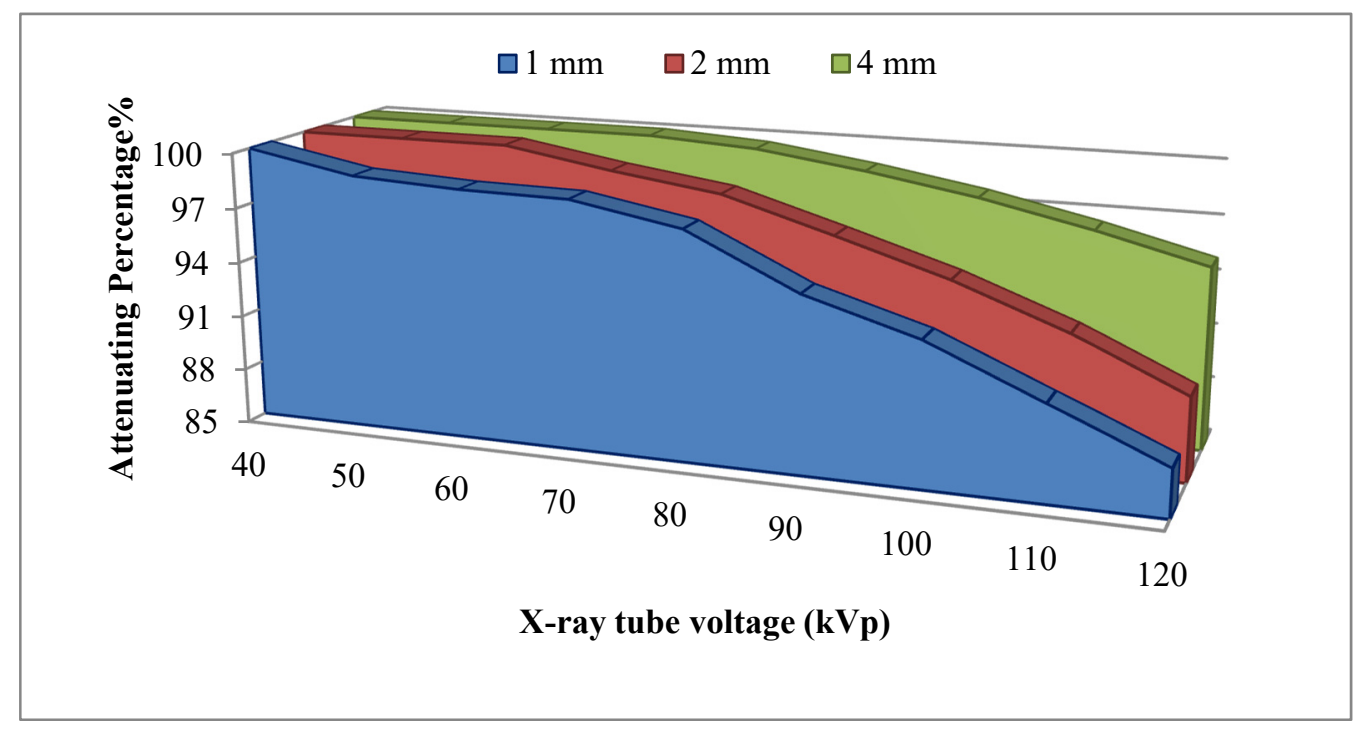

Figure 7. The effect of Iron Steel thickness on attenuation of X-ray

High attenuation percentages for 1-mm thick iron steel can be obtained at low $\mathrm{kVp}(40 \mathrm{kVp})$, whereas penetrating radiation is reduced by approximately $87.6 \%$ at high $\mathrm{kVp}(120 \mathrm{kVp})$.

Figure 7 shows that high $\mathrm{kVp}$ X-ray photons can penetrate the iron steel shield more than low $\mathrm{kVp} \mathrm{X-ray} \mathrm{photons}$ for all iron steel thicknesses. Moreover, the effect of iron steel shield thickness on its capabilities on attenuating incident X-ray is clearly observed in the X-ray and attenuation- $\mathrm{kVp}$ curve. Lower thickness of iron steel $(1 \mathrm{~mm})$ results in steeper slope of the penetrated $\mathrm{X}$-ray percentage curve.

Thus, penetrating radiation decreases with increasing iron steel shield thickness. Higher attenuation of incident Xray occurs at lower $\mathrm{kVp}$ and thicker iron steel shield.

\subsubsection{X-ray Attenuation using Aluminum Shields}

The capability of aluminum shield $\left(\mathrm{Z}=13\right.$, mass density $\left.=2.7 \mathrm{~g} / \mathrm{cm}^{3}\right)$ in attenuating the incident X-ray is also examined. Several measurements are recorded at exposure parameters of $32 \mathrm{mAs}$, FDD of $100 \mathrm{~cm}$, and different $\mathrm{kVp}$ values. Figure 8 shows the capability of $1-\mathrm{mm}$ thick aluminum shield on attenuating the incident X-ray at varying $k V p$ values. 


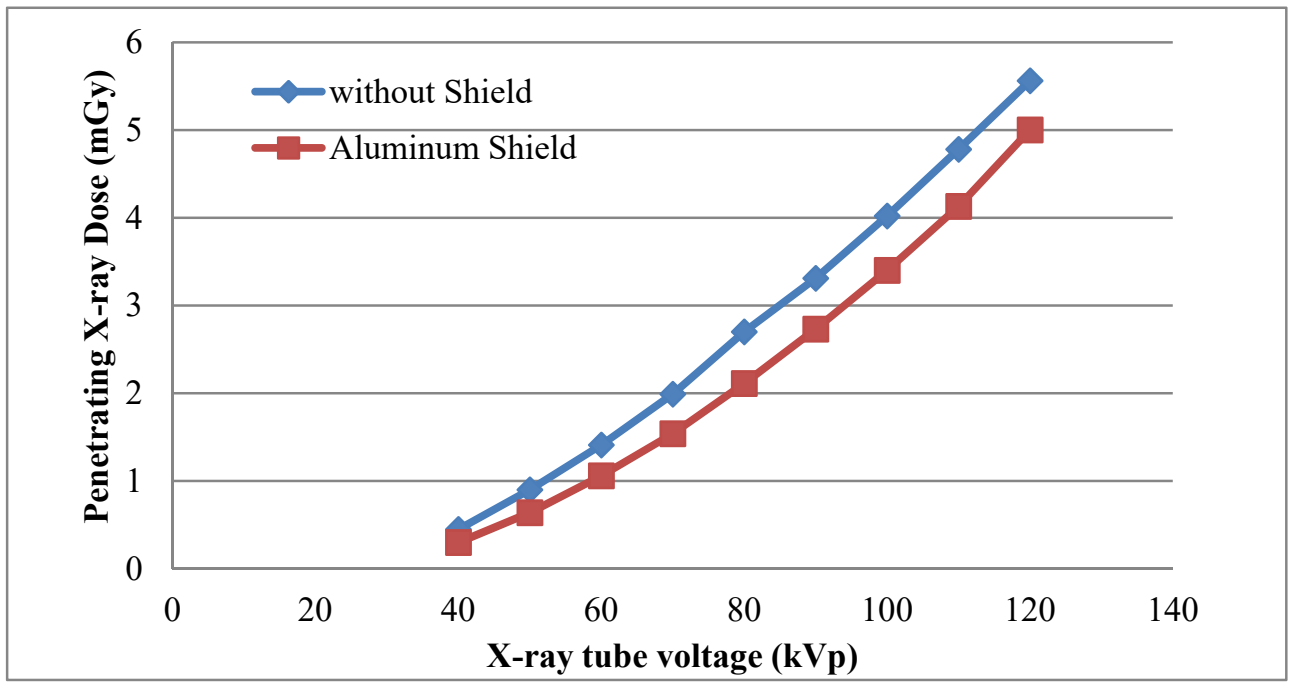

Figure 8. X-ray attenuation by using Aluminum shield

Aluminum has lower attenuating capability compared with the other tested metals because of the composition and density of aluminum material. Contrary to the other metal shields, lower energy of X-ray photons can penetrate the aluminum shield. The effect of aluminum shield thickness on incident X-ray attenuation is illustrated in Figure 9.

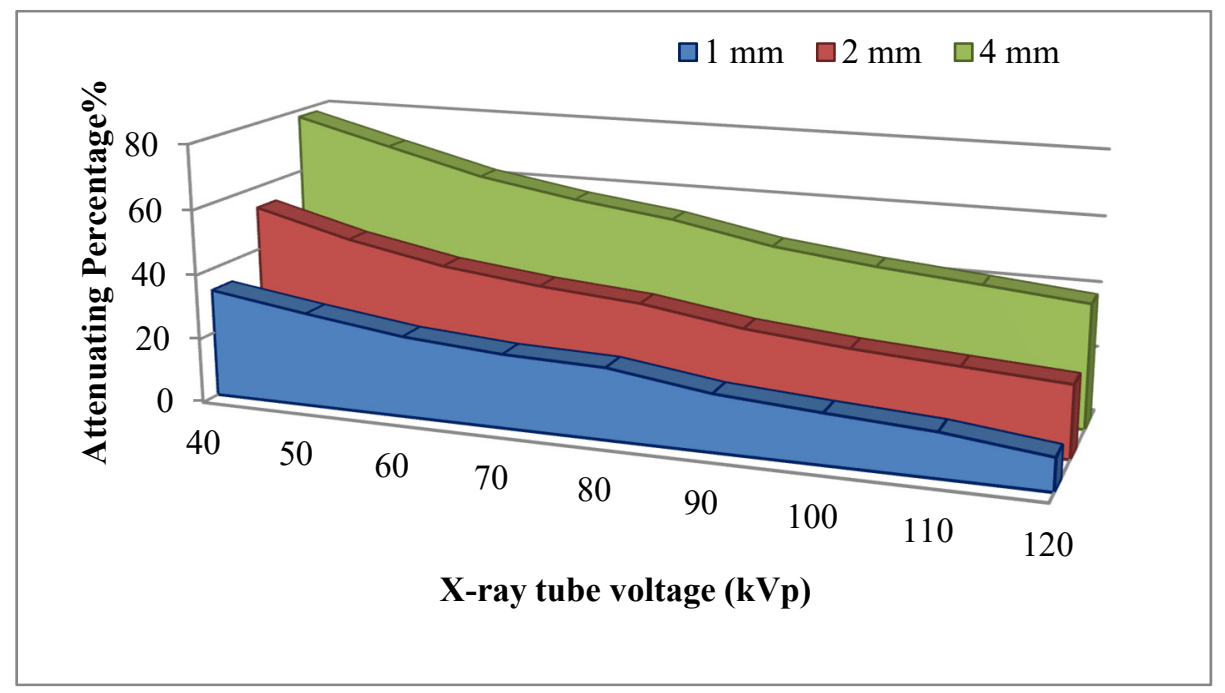

Figure 9. The effect of Aluminum thickness on attenuation of incident X-ray

The percentage of radiation attenuation using 1 -mm thick aluminum is approximately $10.07 \%$ with high X-ray photon energy $(120 \mathrm{kV})$ and reaches $33.33 \%$ for the lowest recorded energy $(40 \mathrm{kVp})$. These values are due to the dependence of X-ray photon penetration on the photon energy and the atomic number of the attenuating medium.

The capability of aluminum in X-ray attenuation is not significant compared with those of the other tested metals. Figure 9 shows direct proportionality between the increasing $\mathrm{kVp}$ and penetrating X-ray dose through aluminum shields. Contrary to other metals, increasing thickness of aluminum shield $(4 \mathrm{~mm})$ results in steeper slope for the radiation attenuation $\mathrm{X}$-ray- $\mathrm{kVp}$ curve.

\subsubsection{Effect of X-rays Intensity (mAs) on the Attenuation of X-ray by Various Metal Shields}

The effect of mAs on the capability of the metal shields in attenuating the incident X-ray is evaluated. Different metals are tested at different mAs values. The measurements are recorded at thickness of $4 \mathrm{~mm}$ for all tested metals, and FDD of $100 \mathrm{~cm}$. The X-ray attenuation percentages are shown in Figure 10. 


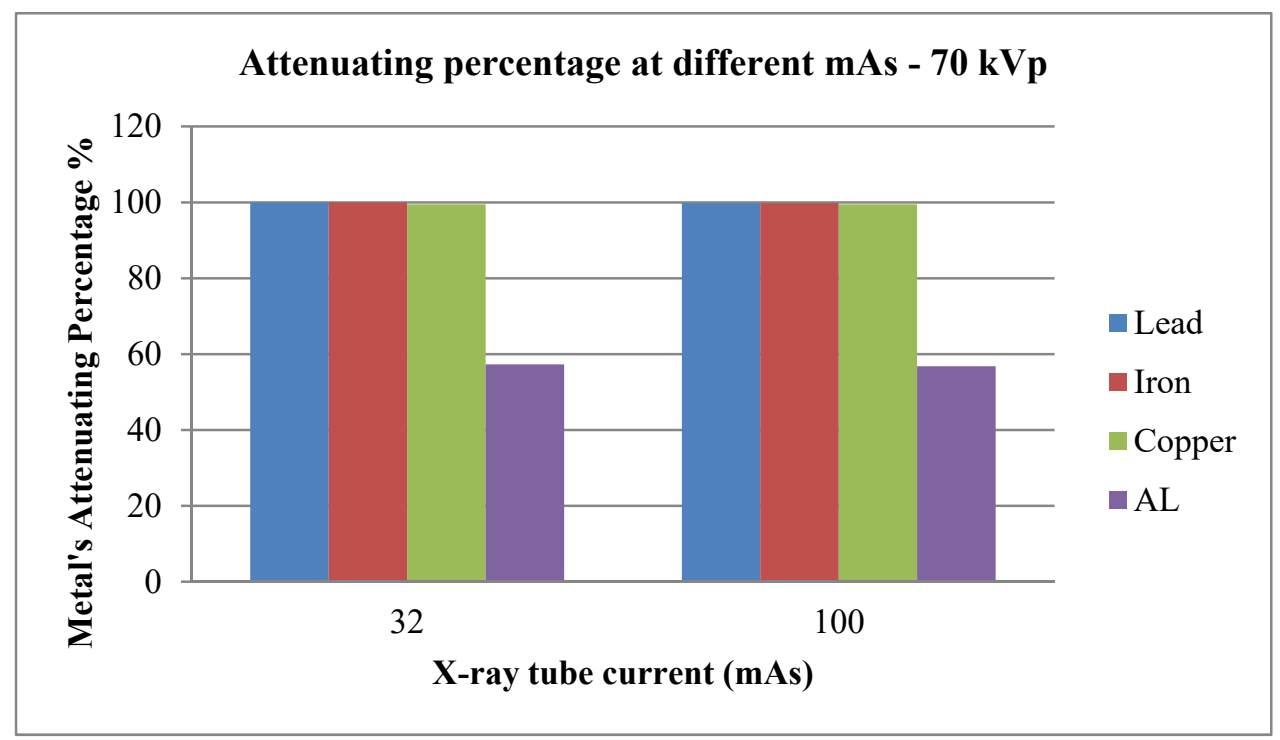

Figure10. X-ray attenuation percentage for several metals at different $\mathrm{mAs}$

No significant difference was found in the attenuation of X-ray related to the variation in mAs. This result refers to the essential dependence of radiation attenuation on the energy of radiation and the material. All measurements for both utilized mAs show that lead, iron steel, and copper are the best matals for X-ray attenuation because of their high densities, whereas aluminum shows the least capability in attenuating $\mathrm{X}$-ray.

The results of section 1 can be summarized as follows: 4-mm thick lead exhibits the best X-ray attenuation for the radiographic energy range of $40 \mathrm{kVp}-120 \mathrm{kVp}$. Iron steel shield exhibits the best values in X-ray attenuation (up to $100 \%$ ) for lower energy photons (less than $50 \mathrm{kVp}$ ). The radiation attenuation percentages reach approximately $99 \%$ and $95 \%$ for $4-\mathrm{mm}$ thick lead and iron shields, respectively, for higher energy photons ( $120 \mathrm{kVp})$. Lead shield is more effective compared with the currently used 4-mm thick cement shield painted with barium sulfate, which had attenuation percentage of up to $95 \%$ (Naji et al., 2015).

The results illustrate the overlap between the atomic number and the mass density, as well as its impact on the attenuation capabilities of the investigated metals.

Moreover, the atomic number is important to determine the capability of a metal for radiation attenuation, whereas $\mathrm{X}$-ray attenuation is caused by both absorption and scattering processes of the primary photons. This phenomenon plays an important role in diagnostic radiology.

Although scattering interaction is independent of atomic number and depends on mass density, more photoelectric interactions occur at high atomic number and high mass density. Thus, the probability of photoelectric effect is directly proportional to the third power of the atomic number of the absorbing material $\left(\mathrm{Z}^{3}\right)$.

Accordingly, the diversity in the attenuation results of the investigated metals refer to the absorption processes (photoelectric effect), and greatly depends on the atomic number and mass density of metals, as well as scatter interactions. These results are in agreement with Bushong (2013) who reported that, the probability for X-ray scattering increases when the mass density of the absorber metal increases, because more electrons are available for the attenuating interactions (scatter and absorption).

The slope of the radiation attenuation percentage versus $\mathrm{kVp}$ curve for each heavy metal tested is dependent on the metal thickness. The thinner the metal thickness, the steeper the slope will be. However, the thicker the aluminum metal, the steeper the slope of the curve will be. Therefore, a non-linear proportionality exists between metal thickness and radiation energy for all tested metals.

The exposure parameters, namely, $\mathrm{kVp}$ and material thickness, affect the attenuating capability of copper and aluminum shields. These result are consistent with the previous published results (Carlsson \& Gudrun, 1996).

The results of X-ray attenuation for the investigated copper and lead metals are dependent on the energy of the Xray and the properties of shield material (including atomic number and mass density), these results are in agreement with earlier findings of Phadnis (2007) and McCaffrey et al. (2007) on copper and lead, respectively. 


\subsection{Effectiveness of Grid's Plate of Tested Metals on Reducing Backscattered Radiation Dose}

The effect of the different metals on the attenuation of backscattered radiation is investigated using these metals plates as base for anti-backscattered iron steel grid. Backscattered dose measurements are recorded at exposure energy of 80 $\mathrm{kVp}$, which are commonly used in radiographic diagnosis as a suggested voltage for non-grid chest X-rays.

The capabilities of anti-backscattered grid with tested metal's plates on reduction of backscattered radiation have evaluated by measuring the backscattered dose with and without placing fabricated grids under exposed object as it illustrated in Figure 1-b. The exposure parameters have been fixed for both cases (with and without utilizing grid and metal's plate). The backscattered results are illustrated in Figure 11.

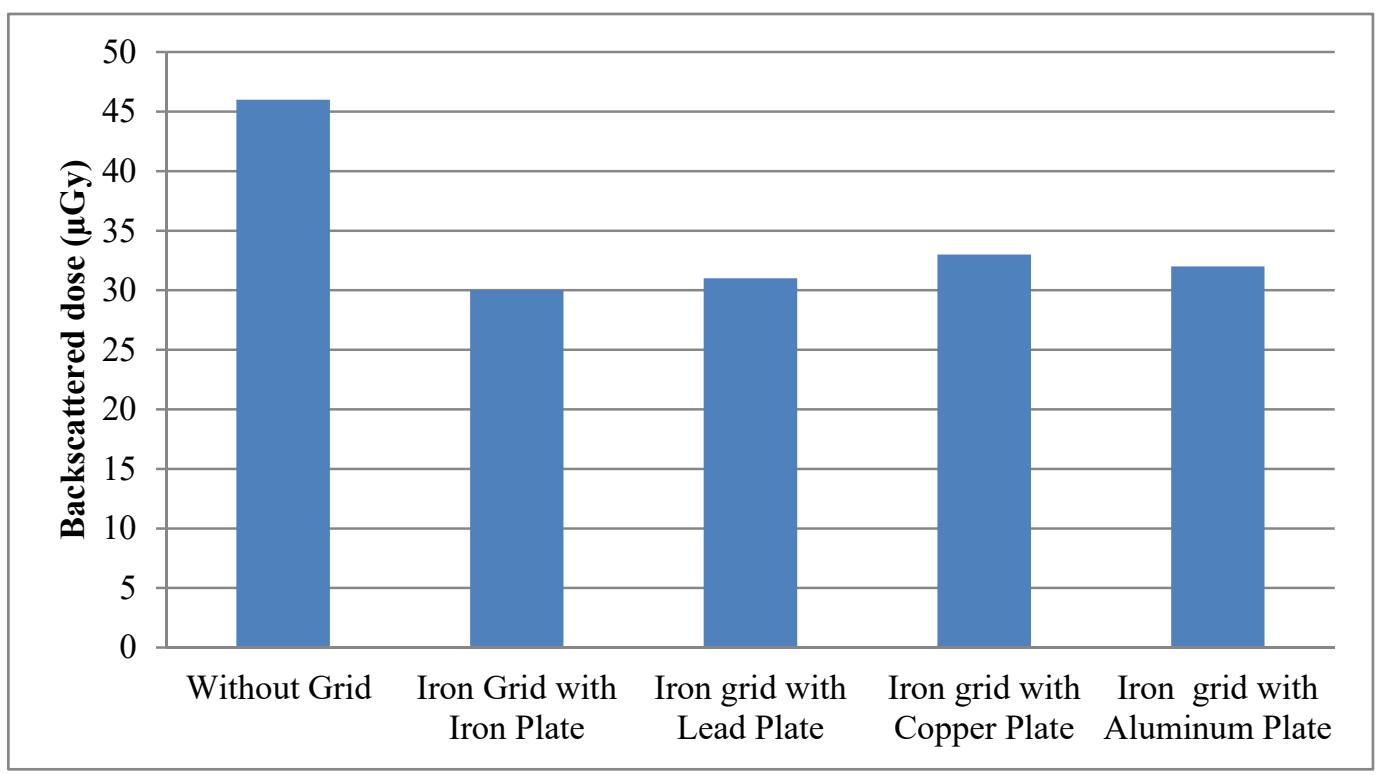

Figure11. Backscattered radiation dose using Iron Grid with different plates

The capability of anti-backscattered iron steel grid is affected using different material types for the grid plate. Differences in the backscattered radiation dose prove the capability of anti-backscattered grids on reducing backscattered dose. The effectiveness of anti-backscattered grids depends on the material of the underlying base of the grid as illustrated in Figure 11. These variations refer to the capability of plate material in absorbing and trapping X-ray photons. The percentages of backscattered radiation reduction are calculated to estimate the effectiveness of the different metal plate in reducing backscattered radiation dose (Figure 12).

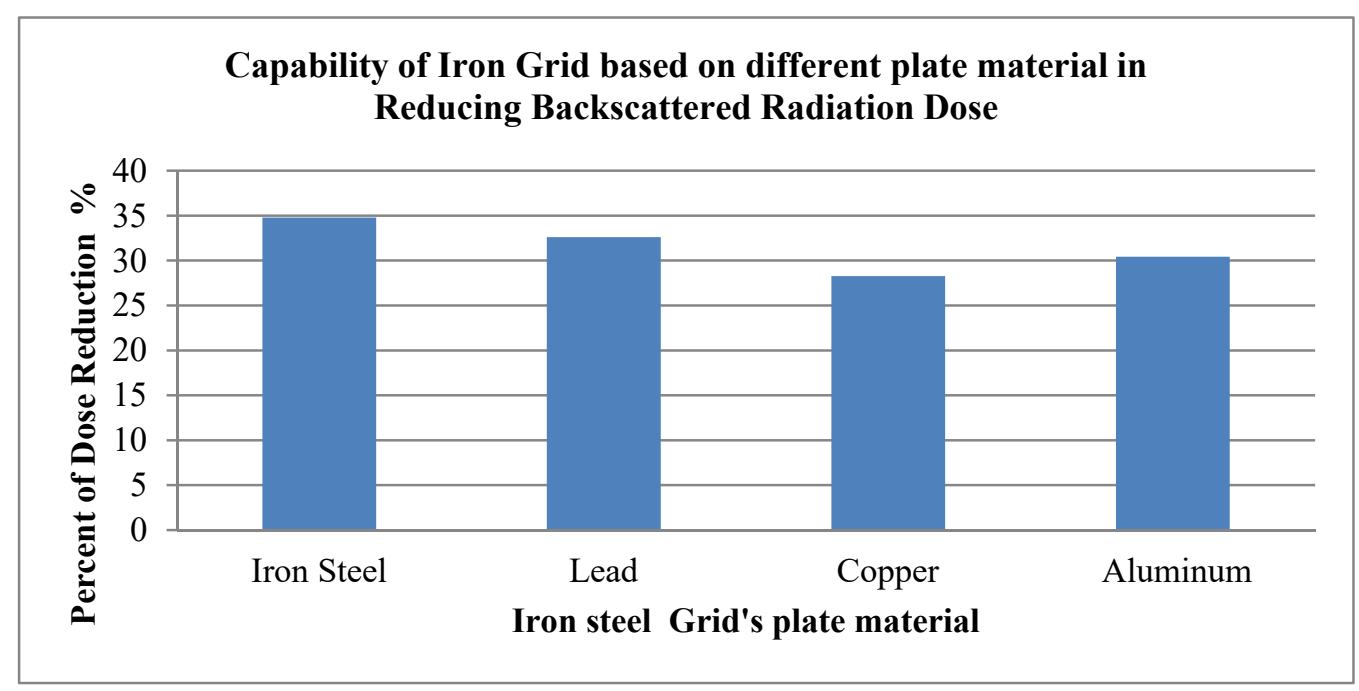

Figure12. Reduction of backscattered radiation percentage for different plate materials of grid 
Backscattered radiation is reduced up to approximately $34.78 \%$ using backscattered grid with iron steel plate. The iron steel plate demonstrates the best ability in reducing backscattered radiation because of its high absorption of the incident X-ray. By contrast, copper plate shows the least ability in reducing backscattered radiation. The differences refer to the capability of metal in absorbing and trapping incident X-ray photons, which reduce the amount of backscattered photons.

Abdul-Majid et al. (2005) reported that iron produces the least amount of backscattered compared with lead and aluminum. The capability of lead in reducing backscattered dose also agrees with the study of Healy et al. (2008) that measured the effect of underlying lead shield on the loss of backscatter for superficial X-ray therapy unit.

Iron steel shield can improve the capability of anti-backscattered grids and protective shields in reducing backscattered radiation during X-ray imaging. This finding offers a new practical method to decrease radiation exposure for patients, staff, and the public inside the X-ray room. Materials used in this work are readily available at a reasonable cost and can easily be shaped and fabricated in various designs for radiation protection shields and anti-scatted radiation grids.

The contribution of this study can be summarized as follows:

First, the capabilities of some metals, namely, lead, iron steel, copper, and aluminum, in attenuating the diagnostic $\mathrm{X}$-ray are evaluated. The attenuation curve behavior of these metals at different exposure settings, materials, and thickness is also illustrated. The evaluated metals can be used to design anti-backscattered grids, X-ray shields, and radiation protection materials.

Second, a procedure to reduce backscattered radiation inside the X-ray room is investigated by estimating the capability of the shield metals, which are used as bases of fabricated anti-backscattered grids to attenuate backscattered X-ray. Backscattered radiation provides the best value in reducing backscattered radiation up to $34.78 \%$.

Third, a method is introduced to measure backscattered radiation dose by a fixed ionization chamber outside the exposed area to measure the backscattered radiation only.

\section{Conclusion}

The capability of materials in attenuating incident and backscattered X-ray photons depends on various factors, such as material density, material components, atomic number, and exposure parameters. Lead, iron steel, aluminum, and copper exhibit different capabilities in attenuating incident and backscattered radiation. Lead and iron steel display the best values in X-ray attenuation. Thus, they can be applied in radiation protection industry. However, iron steel grid with iron plate base is the most effective in reducing backscattered radiation. Iron steel is best-known as the most significant and effective material for eliminating backscattered radiation photons. Nevertheless, further investigations are needed to determine the effect of anti-backscattered radiation grids fabricated from iron steel, lead, aluminum, and copper on the quality of an X-ray image.

\section{References}

Abdulaziz, S. A. (2012). Handbook of quality control in radiography (1st ed.). Malaysia, USM.

Abdul-Majid, S., Kinsara, A., Almasoumi, A., \&Kallothody, M. (2005). Reduction of Backscattered Radiation in Enclosure X-ray Radiography. Presented paper in 3rd MENDT Conference \& Exhibition. 27-30 Nov. 2005.

Aichinger, H., Dierker, J., \&Säbel, M. (2012). Penetration of X-Rays. Radiation Exposure and Image Quality in X-Ray Diagnostic Radiology, Springer.

Blinov, A. (2013). New Requirements for Radiation Protection in X-Ray Surgery. Biomedical Engineering, 46, 212-215.

Bushong, S. (2013). Radiologic Science for Technologists Physics, Biology, and Protection (10th ed.). USA, Elsevier Mosby, Inc.

Carlsson, C.,\&Gudrun, A. (1996). Basic physics of X-ray imaging (2nd ed., pp. 6-9).

Healy, B., S. Sylvander, S., \& Nitshke, K. (2008). Dose reduction from loss of backscatter in superficial x-ray radiation therapy with the Pantak SXT 150 unit. Australasian Physics \& Engineering Sciences in Medicine, $31(1), 49-55$.

Huggett, J., Mukonoweshuro, W., \&Loader, R. (2013). A phantom-based evaluation of three commercially available patient organ shields for computed tomography X-ray examinations in diagnostic radiology. Radiation protection dosimetry, 155, 161-168. 
Kim, E., Han, W., Choi, J., Jung, Y., Yoon, S., \& Lee, J. (2012). Diagnostic reference levels in intraoral dental radiography in Korea. Imaging science in dentistry, 42, 237-242.

Mccaffrey, J., Tessie, F., \&Shen, H. (2012). Radiation shielding materials and radiation scatter effects for interventional radiology (IR) physicians. Medical physics, 39,37-45.

Mccaffrey, J., Shen, H., Downton, B., \&Mainegra, H. (2007). Radiation attenuation by lead and nonlead materials used in radiation shielding garments.Medical Physics, 34(2).

Naji, A.T., Jaafar, M.S., \&Ali, E. (2015). X-ray Protection Using Mixture of Cement Shielding with Barium Sulfate. Journal of Science \& Technology, 20(2), 34-42.

Phadnis, P. (2007). Attenuation of X-Rays in Copper. experiment study. MIT Department of Physics. Retrieved from http://web.mit.edu/8.13/.

Ryu, J., Baek, S., Jung, C., Cho, S., Jung, E., Kim, H., \& Kim, J. (2013). The Survey about the Degree of Damage of Radiation-Protective Shields in Operation Room. The Korean Journal of Pain, 26, 142-147.

\section{Appendix}

Quality control tests of X-ray machine (Images of devices)

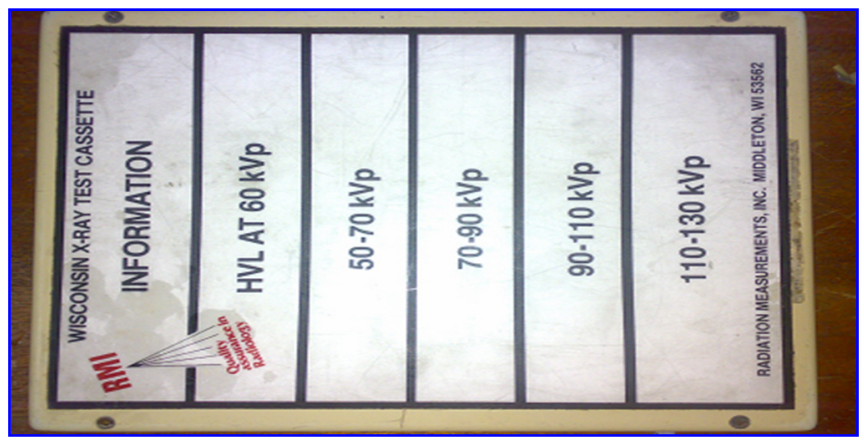

Figure A. kVp and HVL Test Tool (Wisconsin X-ray Test Cassette)

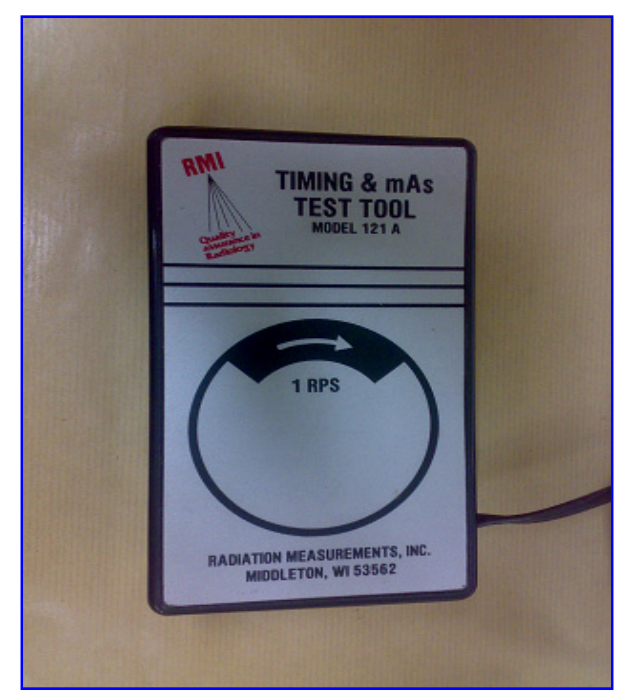

Figure B. Timing and mAs Test Tool (RMI Model 121A)

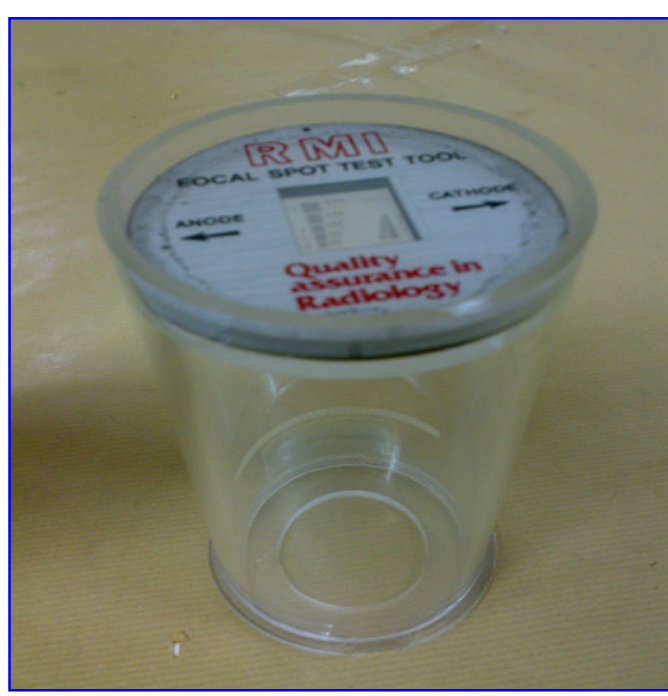

Figure C. Focal Spot Test Tool (RMI Model 112B)

\section{Copyrights}

Copyright for this article is retained by the author(s), with first publication rights granted to the journal.

This is an open-access article distributed under the terms and conditions of the Creative Commons Attribution license (http://creativecommons.org/licenses/by/4.0/). 